\title{
DEPLETION OF NONLINEARITY IN THE PRESSURE FORCE DRIVING NAVIER-STOKES FLOWS
}

\author{
CHUONG V. TRAN AND XINWEI YU
}

\begin{abstract}
The dynamics of the velocity norms $\|u\|_{L^{q}}$, for $q \geq 3$, in Navier-Stokes flows is studied. The pressure term that drives this dynamics has a high degree of nonlinear depletion, which owes its origin to a genuine negative correlation between $|u|$ and $|\nabla| u||$, among other things. Under viscous effects, such depletion may give rise to mild growth of $\|u\|_{L^{q}}$. We explore the possibility of non-singular growth of $\|u\|_{L^{q}}$.
\end{abstract}

\section{INTRODUCTION}

A long-standing issue in mathematical fluid dynamics is concerned with whether solutions of the three-dimensional Navier-Stokes equations evolving from smooth (but otherwise arbitrary) initial velocity fields remain globally smooth (regular) in time. This problem is fundamentally important and of both theoretical and practical interest. Decades of active research since Leray's seminal studies in the 1930s have resulted in a rich literature $[3,4,7,8,9,10,11,12,18,19,20,22,23,26$, $27,28,29,33,36,37,38,39,42]$. Yet, the prospect of a definitive answer to the problem remains remote, leading to its designation as one of the millennium prize problems by the Clay Mathematics Institute.

Existing results to date on Navier-Stokes regularity are mainly in the form of conditional regularity. Early studies by Prodi [33], Serrin [36] and Ladyzhenskaya [28] found that regularity (up to some time $t$ ) is guaranteed provided that $\int_{0}^{t}\|u\|_{L^{q}}^{2 q /(q-3)} d \tau<\infty$, for $q>3$. Here $u$ is the fluid velocity and $\|\cdot\|_{L^{q}}$ denotes the usual norm in the Lebesque spaces $L^{q}$. Recently, this criterion was extended to $\|u\|_{L^{3}}<\infty$, for the limiting case $q=3$ [18]. On the basis of these results, various criteria expressible in terms of the pressure $p$ and its gradient $\nabla p$ have been derived by a number of authors $[5,10,32,41,42]$. One of the main results along this line of research is the criterion $\int_{0}^{t}\|p\|_{L^{\alpha}}^{2 \alpha /(2 \alpha-3)} \mathrm{d} \tau<\infty$, for $\alpha \in(3 / 2, \infty)$, representing some moderate improvement of the ProdiSerrin-Ladyzhenskaya result. From a quite different approach, Seregin and Sverák [35] proved that no singularities can develop if $p$ ("normalized" to zero at infinity) is bounded from below. The theory of Navier-Stokes regularity has also been enriched by a wealth of criteria expressible in terms of one component of $u$ or $\nabla u[8,9,26,27,40,43,46,47]$, the velocity direction $u /|u|[11,29,39]$ or vorticity direction $\omega /|\omega|[3,4,12,20,44,45]$.

In a parallel development, a number of regularity criteria have been derived for the Euler equations $[2,13,15,16,25]$. However, the results are suboptimally applicable or even inapplicable to the Navier-Stokes case. On the one hand, criteria based on energy-type estimates, such as the

Date: February 16, 2015.

2000 Mathematics Subject Classification. 76D03,76D05.

Key words and phrases. Navier-Stokes equations, Momentum growth, Depletion of nonlinearity, Global regularity. 
Beale-Kato-Majda [2] criterion $\int_{0}^{t}\|\omega\|_{L^{\infty}} d \tau<\infty$ and the Constantin-Fefferman-Majda [13] criterion, associating the smoothness of the vorticity direction $\omega /|\omega|$ with regularity, can be improved to a great extent by incorporating viscous effects. On the other hand, the Deng-Hou-Yu $[15,16]$ criterion, which is based on local analysis of "frozen" vortex lines, becomes inapplicable in the presence of viscosity as the vortex lines are no longer frozen in the flow.

Given the apparent inadequacy of viscous effects in regularising Navier-Stokes flows [38], researchers have looked for the possibility of nonlinear depletion in the vortex dynamics. Such depletion is a well-documented phenomenon for both Euler and Navier-Stokes cases [1, 6, 17, 21, 24, $30,31,34]$. The question is how to capture and exploit this favourable phenomenon in a rigorous manner. A recent investigation by Donzis et al. [17] has addressed this question in some detail.

In this study, we examine possible nonlinear depletion in the momentum dynamics, i.e. in the pressure term of the governing equation for $\|u\|_{L^{q}}$, for $q \geq 3$. Our focus is on the genuine negative correlation between the factors $|u|$ and $|\nabla| u||$ within this term. Such a correlation can give rise to a certain degree of nonlinear depletion, possibly allowing for mild growth of $\|u\|_{L^{q}}$ under viscous effects. We explore this possibility by elementary methods and derive some new regularity criteria.

\section{Dynamics of $\|u\|_{L^{q}}$ AND Basic estimates}

2.1. Preliminaries. The Navier-Stokes equations governing the motion of a viscous and incompressible fluid are

$$
\begin{aligned}
\frac{\partial u}{\partial t}+(u \cdot \nabla) u+\nabla p & =\Delta u, \\
\nabla \cdot u & =0, \\
u(x, 0) & =u_{0}(x),
\end{aligned}
$$

where, again, $u$ is the fluid velocity, $p$ is the pressure and the kinematic viscosity coefficient has been set to unity for convenience. The fluid is assumed to fill all $\mathbb{R}^{3}$ with the usual condition of sufficient decay at infinity.

Given a smooth (but otherwise arbitrary) initial velocity field $u(x, 0)=u_{0}(x)$, equation (1) is known to admit a smooth and unique solution locally in time. This means that the task of proving global regularity is reduced to the removal this locality, thus extending the validity of the local solution to all $t<\infty$. In the search for such an extension, one may therefore employ usual manipulations of (1) in the classical sense without restriction. This study assumes smooth $u_{0}(x)$ throughout. All constants, which may vary from one expression to another, are denoted by $c$.

By taking the divergence of the momentum equation in (1) and by virtue of $\nabla \cdot u=0$, one obtains

$$
\Delta p=-\nabla \cdot((u \cdot \nabla) u)=-\sum_{i, j=1}^{3} \partial_{i} \partial_{j}\left(u_{i} u_{j}\right)
$$

The Calderon-Zygmund inequality implies

$$
\|p\|_{L^{q}} \leq c\|u\|_{L^{2 q}}^{2}, \text { for } 1<q<\infty .
$$

Taking the gradient of (2) yields

$$
\Delta \nabla p=-\sum_{i, j=1}^{3} \partial_{i} \partial_{j}\left(\nabla\left(u_{i} u_{j}\right)\right) .
$$


Again by Calderon-Zygmund's inequality one has

$$
\|\nabla p\|_{L^{q}} \leq c\||u| \nabla u\|_{L^{q}}, \text { for } 1<q<\infty .
$$

Equations (3) and (5) put $p$ on an equal footing with $|u|^{2}$.

The evolution of the local energy $|u|^{2} / 2$ is governed by

$$
\frac{\partial}{\partial t} \frac{|u|^{2}}{2}+u \cdot \nabla \frac{|u|^{2}}{2}+u \cdot \nabla p=\Delta \frac{|u|^{2}}{2}-|\nabla u|^{2} .
$$

Integrating (6) over the domain yields the equation governing the decay of the global energy $\|u\|_{L^{2}}^{2} / 2$ :

$$
\frac{1}{2} \frac{\mathrm{d}}{\mathrm{d} t}\|u\|_{L^{2}}^{2}=-\|\nabla u\|_{L^{2}}^{2}=-\|\omega\|_{L^{2}}^{2}
$$

where the integrals of the advection, pressure and Laplacian terms identically vanish. These terms redistribute energy in space, but with distinct effects. As far as the issue of regularity is concerned, the advection term merely transport energy (and momentum), therefore having neutral consequences. The pressure term accelerates and decelerates fluid particles, allowing for the possibility of extreme energy and momentum concentration, i.e. for possible singularity development. The Laplacian term tends to homogenize the energy, thereby counteracting the "focusing" effect of the pressure. A detailed knowledge of the opposing tendencies of these two terms is key to understanding the issue of solution regularity. Note that the negative definite term $-|\nabla u|^{2}$ locally dissipates energy and certainly renders favourable effects on regularity.

Integrating (7) over time yields the bound

$$
\int_{0}^{t}\|\omega\|_{L^{2}}^{2} \mathrm{~d} \tau \leq \frac{\left\|u_{0}\right\|_{L^{2}}^{2}}{2}
$$

This result is one of a few exact estimates derivable from first principles and is not known to have adequate smoothness to guarantee global existence of smooth solutions (the integrability of $\|\omega\|_{L^{2}}^{4}$ over time is more than sufficient as demonstrated in $\S 3$ ). Other such estimates include (3), (5) and $\|u\|_{L^{2}} \leq\left\|u_{0}\right\|_{L^{2}}$.

2.2. Bounds for $\|u\|_{L^{q}}$. Consider $q \geq 3$. Multiplying (6) by $|u|^{q-2}$ and integrating the resulting equation over the domain yield

$$
\begin{aligned}
\|u\|_{L^{q}}^{q-1} \frac{\mathrm{d}}{\mathrm{d} t}\|u\|_{L^{q}} & =-\int_{\mathbb{R}^{3}}|u|^{q-2} u \cdot \nabla p \mathrm{~d} x+\int_{\mathbb{R}^{3}}|u|^{q-2}\left(\Delta \frac{|u|^{2}}{2}-|\nabla u|^{2}\right) \mathrm{d} x \\
& =(q-2) \int_{\mathbb{R}^{3}} p|u|^{q-3} u \cdot \nabla|u| \mathrm{d} x-\left.(q-2) \int_{\mathbb{R}^{3}}|u|^{q-2}|\nabla| u\right|^{2} \mathrm{~d} x-\int_{\mathbb{R}^{3}}|u|^{q-2}|\nabla u|^{2} \mathrm{~d} x \\
& \leq(q-2) \int_{\mathbb{R}^{3}}|p||u|^{q-2}|\nabla| u|| \mathrm{d} x-(q-2) \int_{\mathbb{R}^{3}}|u|^{q-2}|\nabla| u||^{2} \mathrm{~d} x-\int_{\mathbb{R}^{3}}|u|^{q-2}|\nabla u|^{2} \mathrm{~d} x \\
& \leq(q-2) \int_{\mathbb{R}^{3}} p^{2}|u|^{q-2} \mathrm{~d} x-\int_{\mathbb{R}^{3}}|u|^{q-2}|\nabla u|^{2} \mathrm{~d} x
\end{aligned}
$$

In (9), the advection term vanishes due to the incompressibility condition, the pressure term and one of the dissipation terms have been obtained by integration by parts, the penultimate inequality is straightforward and the final line is due to Young's inequality. By omitting the negative term on 
the right-hand side of (9) we obtain

$$
\frac{\mathrm{d}}{\mathrm{d} t}\|u\|_{L^{q}} \leq \frac{q-2}{\|u\|_{L^{q}}^{q-1}} \int_{\mathbb{R}^{3}} p^{2}|u|^{q-2} \mathrm{~d} x \leq \frac{(q-2)\|p\|_{L^{q}}^{2}\|u\|_{L^{q}}^{q-2}}{\|u\|_{L^{q}}^{q-1}}=\frac{(q-2)\|p\|_{L^{q}}^{2}}{\|u\|_{L^{q}}^{2}}\|u\|_{L^{q}},
$$

where Hölder's inequality has been used. By direct integration of (10) over time (Gronwall's lemma), we obtain the following criterion.

Theorem 1. Let $u$ and $p$ solve the Navier-Stokes equations (1). If

$$
\int_{0}^{t} \frac{\|p\|_{L^{q}}^{2}}{\|u\|_{L^{q}}^{2}} \mathrm{~d} \tau<\infty
$$

then

$$
\|u\|_{L^{q}} \leq\left\|u_{0}\right\|_{L^{q}} \exp \left\{(q-2) \int_{0}^{t} \frac{\|p\|_{L^{q}}^{2}}{\|u\|_{L^{q}}^{2}} \mathrm{~d} \tau\right\}<\infty
$$

and regularity follows.

It is worth mentioning that a straightforward incorporation of a factor of $\ln \left(e+\|u\|_{L^{q}}\right)$ into the evolution equation (10) results in the appearance of such a factor in the denominator of the integrand in (11). We have omitted this marginal improvement for simplicity. Such an improvement will be omitted throughout this study.

Criterion (11) can be written in terms of $u$ alone thanks to (3). Indeed, a straightforward substitution of (3) into (10) yields the following corollary.

Corollary 1. If

$$
\int_{0}^{t} \frac{\|u\|_{L^{2 q}}^{4}}{\|u\|_{L^{q}}^{2}} \mathrm{~d} \tau<\infty
$$

then

$$
\|u\|_{L^{q}} \leq\left\|u_{0}\right\|_{L^{q}} \exp \left\{c(q-2) \int_{0}^{t} \frac{\|u\|_{L^{2 q}}^{4}}{\|u\|_{L^{q}}^{2}} \mathrm{~d} \tau\right\}<\infty
$$

and regularity follows.

For $q=3$, one can substitute (3) and the Sobolev inequality $\|u\|_{L^{6}} \leq c\|\omega\|_{L^{2}}$ into (10) and obtain the following regularity criterion.

Corollary 2. If

$$
\int_{0}^{t} \frac{\|\omega\|_{L^{2}}^{4}}{\|u\|_{L^{3}}^{2}} \mathrm{~d} \tau<\infty
$$

then

$$
\|u\|_{L^{q}} \leq\left\|u_{0}\right\|_{L^{q}} \exp \left\{c(q-2) \int_{0}^{t} \frac{\|\omega\|_{L^{2}}^{4}}{\|u\|_{L^{3}}^{2}} \mathrm{~d} \tau\right\}<\infty
$$

and regularity follows. 
This result improves the well-known criterion $\int_{0}^{t}\|\omega\|_{L^{2}}^{4} \mathrm{~d} \tau<\infty$ by a factor of $\|u\|_{L^{3}}^{-2}$.

A criterion slightly different from (13) can be derived by considering the evolution equation for $\|u\|_{L^{4}}$. By substituting $q=4$ into (9), we obtain

$$
\begin{aligned}
\|u\|_{L^{4}}^{3} \frac{\mathrm{d}}{\mathrm{d} t}\|u\|_{L^{4}} & =-\int_{\mathbb{R}^{3}}|u|^{2} u \cdot \nabla p \mathrm{~d} x+\int_{\mathbb{R}^{3}}|u|^{2}\left(\Delta \frac{|u|^{2}}{2}-|\nabla u|^{2}\right) \mathrm{d} x \\
& \leq \int_{\mathbb{R}^{3}}|u|^{3}|\nabla p| \mathrm{d} x-\left.2 \int_{\mathbb{R}^{3}}|u|^{2}|\nabla| u\right|^{2} \mathrm{~d} x-\int_{\mathbb{R}^{3}}|u|^{2}|\nabla u|^{2} \mathrm{~d} x \\
& \leq\|u\|_{L^{6}}^{3}\|\nabla p\|_{L^{2}}-\left.2 \int_{\mathbb{R}^{3}}|u|^{2}|\nabla| u\right|^{2} \mathrm{~d} x-\||u| \nabla u\|_{L^{2}}^{2} \\
& \leq c\|u\|_{L^{6}}^{6}-2 \int_{\mathbb{R}^{3}}|u|^{2}|\nabla| u \|^{2} \mathrm{~d} x,
\end{aligned}
$$

where Hölder's inequality, (5) and Young's inequality have been used. It follows that

$$
\frac{\mathrm{d}}{\mathrm{d} t}\|u\|_{L^{4}} \leq c \frac{\|u\|_{L^{6}}^{6}}{\|u\|_{L^{4}}^{3}}=c \frac{\|u\|_{L^{6}}^{6}}{\|u\|_{L^{4}}^{4}}\|u\|_{L^{4}} .
$$

Hence we have the following criterion upon integration.

Theorem 2. Let $u$ and $p$ solve the Navier-Stokes equations (1). If

$$
\int_{0}^{t} \frac{\|u\|_{L^{6}}^{6}}{\|u\|_{L^{4}}^{4}} \mathrm{~d} \tau<\infty
$$

then

$$
\|u\|_{L^{4}} \leq\left\|u_{0}\right\|_{L^{4}} \exp \left\{c \int_{0}^{t} \frac{\|u\|_{L^{6}}^{6}}{\|u\|_{L^{4}}^{4}} \mathrm{~d} \tau\right\}
$$

and regularity follows.

By invoking the Sobolev inequality, this result implies a further corollary.

Corollary 3. If

$$
\int_{0}^{t} \frac{\|\omega\|_{L^{2}}^{6}}{\|u\|_{L^{4}}^{4}} \mathrm{~d} \tau<\infty
$$

then

$$
\|u\|_{L^{4}} \leq\left\|u_{0}\right\|_{L^{4}} \exp \left\{c \int_{0}^{t} \frac{\|\omega\|_{L^{2}}^{6}}{\|u\|_{L^{4}}^{4}} \mathrm{~d} \tau\right\}
$$

and regularity follows.

It is desirable to reduce $q$ in the norm $\|p\|_{L^{q}}$ in (11). Such a reduction is possible with a reasonable price. In what follows, we derive yet another family of criteria expressible in terms of $\|p\|_{L^{3 q /(q+1)}}$.

Theorem 3. Let $u$ and $p$ solve the Navier-Stokes equations (1). If

$$
\int_{0}^{t} \frac{\|p\|_{L^{3 q /(q+1)}}^{q}}{\|u\|_{L^{q}}^{q}} \mathrm{~d} \tau<\infty
$$


then

$$
\|u\|_{L^{q}} \leq\left\|u_{0}\right\|_{L^{q}} \exp \left\{c(q-2)^{q / 2} q^{q-2} \int_{0}^{t} \frac{\|p\|_{L^{3 q /(q+1)}}^{q}}{\|u\|_{L^{q}}^{q}} \mathrm{~d} \tau\right\}
$$

and regularity follows.

Proof. From the penultimate step in (9) we have

$$
\begin{aligned}
\|u\|_{L^{q}}^{q-1} \frac{\mathrm{d}}{\mathrm{d} t}\|u\|_{L^{q}} \leq & (q-2) \int_{\mathbb{R}^{3}}|p||u|^{q-2}|\nabla| u \| \mathrm{d} x-\left.(q-2) \int_{\mathbb{R}^{3}}|u|^{q-2}|\nabla| u\right|^{2} \mathrm{~d} x-\int_{\mathbb{R}^{3}}|u|^{q-2}|\nabla u|^{2} \mathrm{~d} x \\
\leq & (q-2)\|p\|_{L^{\alpha}}\left\||u|^{(q-2) / 2}\right\|_{L^{\beta}}\left\||u|^{(q-2) / 2} \nabla|u|\right\|_{L^{2}} \\
& -(q-2)\left\|\left.|u|^{(q-2) / 2} \nabla\left|u\left\|_{L^{2}}^{2}-\right\|\right| u\right|^{(q-2) / 2} \nabla u\right\|_{L^{2}}^{2} \\
\leq & (q-2)\|p\|_{L^{\alpha}}^{2}\left\||u|^{(q-2) / 2}\right\|_{L^{\beta}}^{2}-\left\||u|^{(q-2) / 2} \nabla u\right\|_{L^{2}}^{2}
\end{aligned}
$$

where Hölder's inequality with exponents $\alpha, \beta$ and 2 satisfying $1 / \alpha+1 / \beta+1 / 2=1$ and Young's inequality have been used. To make use of the remaining dissipation term in (25) we observe that $\nabla|u|^{q / 2}=q|u|^{(q-2) / 2} \nabla|u| / 2$. So

$$
\begin{aligned}
\left\||u|^{(q-2) / 2} \nabla u\right\|_{L^{2}} & \geq\left\||u|^{(q-2) / 2} \nabla|u|\right\|_{L^{2}}=\frac{2}{q}\left\|\nabla|u|^{q / 2}\right\|_{L^{2}} \\
& \geq \frac{c}{q}\left\||u|^{q / 2}\right\|_{L^{6}}=\frac{c}{q}\|u\|_{L^{3 q}}^{q / 2},
\end{aligned}
$$

where the Sobolev inequality has been used. Now let $\beta=6 q /(q-2)$, then $\alpha=3 q /(q+1)$ and $\left\||u|^{(q-2) / 2}\right\|_{L^{\beta}}=\|u\|_{L^{3 q}}^{(q-2) / 2}$. By substituting these results and (26) into (25) we obtain

$$
\begin{aligned}
\|u\|_{L^{q}}^{q-1} \frac{\mathrm{d}}{\mathrm{d} t}\|u\|_{L^{q}} & \leq(q-2)\|p\|_{L^{3 q /(q+1)}}^{2}\|u\|_{L^{3 q}}^{q-2}-\frac{c}{q^{2}}\|u\|_{L^{3 q}}^{q} \\
& \leq c(q-2)^{q / 2} q^{q-2}\|p\|_{L^{3 q /(q+1)}}^{q} \\
& =c(q-2)^{q / 2} q^{q-2}\|p\|_{L^{3 q /(q+1)}}^{q}
\end{aligned}
$$

where Young's inequality has been used. The theorem is proven upon integrating (27).

Clearly, the norm $\|p\|_{L^{3 q /(q+1)}}$ in (23) is much weaker than $\|p\|_{L^{q}}$ in (11). However, this does not necessarily mean that (23) is an improvement of (11) for all $q$ because of the high discrepancy in the power of these norms. The same can be said of the criterion $\int_{0}^{t}\|p\|_{L^{\alpha}}^{2 \alpha /(2 \alpha-3)} \mathrm{d} \tau<\infty$, for $\alpha \in(3 / 2, \infty)$ mentioned earlier.

\section{Nonlinear Depletion And REFined BOUnds FOR $\|u\|_{L^{q}}$}

3.1. Nonlinear depletion in vortex stretching: a heuristic argument. By taking the curl of the momentum equation one obtains the vorticity equation

$$
\frac{\partial \omega}{\partial t}+(u \cdot \nabla) \omega=(\omega \cdot \nabla) u+\Delta \omega
$$

The evolution of $\|\omega\|_{L^{2}}^{2}$ is governed by

$$
\frac{1}{2} \frac{\mathrm{d}}{\mathrm{d} t}\|\omega\|_{L^{2}}^{2}=\int_{\mathbb{R}^{3}} \omega \cdot(\omega \cdot \nabla) u \mathrm{~d} x-\|\nabla \omega\|_{L^{2}}^{2} .
$$


Suppose that

$$
\int_{\mathbb{R}^{3}} \omega \cdot(\omega \cdot \nabla) u \mathrm{~d} x \leq C \int_{\mathbb{R}^{3}}|\omega|^{3-\theta} \mathrm{d} x=C\|\omega\|_{L^{3-\theta}}^{3-\theta},
$$

where $C(t)$ is a bounded function (which can vary from one expression to another in what follows) of suitable dimension and $0 \leq \theta \leq 1$. Here, $\theta$ can be thought of as a measure of nonlinear depletion, with $\theta=1$ and $\theta=0$ corresponding to complete and no depletion, respectively. By using Gagliardo-Nirenberg's inequality we can write

$$
\|\omega\|_{L^{3-\theta}}^{3-\theta} \leq c\|\omega\|_{L^{2}}^{(3+\theta) / 2}\|\nabla \omega\|_{L^{2}}^{3(1-\theta) / 2} .
$$

Substituting this estimate into (29) yields

$$
\frac{1}{2} \frac{\mathrm{d}}{\mathrm{d} t}\|\omega\|_{L^{2}}^{2} \leq C\|\omega\|_{L^{2}}^{(3+\theta) / 2}\|\nabla \omega\|_{L^{2}}^{3(1-\theta) / 2}-\|\nabla \omega\|_{L^{2}}^{2} \leq C\|\omega\|_{L^{2}}^{2(3+\theta) /(1+3 \theta)}
$$

where Young's inequality has been used. Consider $\theta=1 / 5$, equation (32) then becomes

$$
\frac{\mathrm{d}}{\mathrm{d} t}\|\omega\|_{L^{2}} \leq C\|\omega\|_{L^{2}}^{3}
$$

It follows that

$$
\|\omega\|_{L^{2}} \leq\left\|\omega_{0}\right\|_{L^{2}} \exp \left\{\int_{0}^{t} C\|\omega\|^{2} \mathrm{~d} \tau\right\}<\infty
$$

and regularity is secured. Note that the same conclusion holds for $\theta>1 / 5$. Hence, a nonlinear depletion in the vortex stretching term represented by (30) with $\theta \geq 1 / 5$ is adequate for regularity.

Donzis et al. [17] have numerically observed the constraint $\|\omega\|_{L^{m+2}}^{(m+2) /(2 m+1)}<c\|\omega\|_{L^{m}}^{m /(2 m-3)}$, for $m=2,4,6, \cdots$. These authors have attributed this observation to a depletion of nonlinearity that could be the cause of regularity. Evidently, the constraint amounts to the decreasingly ordered set $\left\{\|\omega\|_{L^{2}}^{2},\|\omega\|_{L^{4}}^{4 / 5},\|\omega\|_{L^{6}}^{2 / 3},\|\omega\|_{L^{8}}^{8 / 13}, \cdots\right\}$, up to constant factors. It is, however, not obvious how this could be used to rule out the possibility of singularity development.

3.2. Nonlinear depletion in pressure force. Unlike the vortex stretching term, for which no "sources" responsible for nonlinear depletion can be readily identified, the pressure term has a number of obvious features capable of nonlinear depletion. These features are the subject of analysis for the remainder of this study. Recall the second line of (9):

$$
\begin{aligned}
\|u\|_{L^{q}}^{q-1} \frac{\mathrm{d}}{\mathrm{d} t}\|u\|_{L^{q}}= & (q-2) \int_{\mathbb{R}^{3}} p|u|^{q-2} \widehat{u} \cdot \nabla|u| \mathrm{d} x-\left.(q-2) \int_{\mathbb{R}^{3}}|u|^{q-2}|\nabla| u\right|^{2} \mathrm{~d} x \\
& -\int_{\mathbb{R}^{3}}|u|^{q-2}|\nabla u|^{2} \mathrm{~d} x
\end{aligned}
$$

where $\widehat{u}=u /|u|$. As it stands, the pressure term in (35) possesses several features that can weaken its nonlinearity. One is that the global maximum of $|u|$ is coupled with the global minimum of $|\nabla| u||$. This negative correlation (also known as anti-correlation) between $|u|$ and $|\nabla| u||$ moderates the contribution from a neighbourhood of peak $|u|$ to the pressure term. Another is that for pure shear flows, the equation $\widehat{u} \cdot \nabla|u|=0$ holds throughout, implying that regions of strong shear (including those with intense vortices) may not make predominant contributions to the pressure term. Furthermore, in a neighbourhood of the global maximum of $p,|u|$ is moderate because inflow (flow toward peak $p$ ) and outflow are decelerated and weakly accelerated, respectively. Hence, such a neighbourhood has a relatively mild contribution to the pressure term. Finally, in the vicinity of 
the global minimum of $p$ (negative), we expect $\widehat{u} \cdot \nabla|u|>0$ for inflow, so that $p \widehat{u} \cdot \nabla|u|<0$. Since outflow is decelerated, such vicinity is likely to make a mild (or even negative) contribution to the pressure term.

It is not clear how to turn the above phenomenology into a quantitative theory. Nevertheless, it is obvious that decoupling the factors involved in the pressure term by standard inequalities necessarily destroys the favourable correlations among these factors. With this in mind we consider the following approach.

Given $r \geq 1 / 2$, let $U_{r}(t)$ be defined by

$$
U_{r}^{r}=\frac{\int_{\mathbb{R}^{3}}|u|^{r} p|u|^{r} \widehat{u} \cdot \nabla|u| \mathrm{d} x}{\|p\|_{L^{2}}\left\||u|^{r} \nabla|u|\right\|_{L^{2}}}=\int_{\mathbb{R}^{3}}|u|^{r} W_{r} d x,
$$

where

$$
W_{r}(x, t)=\frac{p|u|^{r} \widehat{u} \cdot \nabla|u|}{\|p\|_{L^{2}}\left\||u|^{r} \nabla|u|\right\|_{L^{2}}} .
$$

Here the "weight function" $W_{r}(x, t)$ has all the favourable features described in the preceding paragraphs and satisfies $\left\|W_{r}\right\|_{L^{1}} \leq 1$. The "weighted norm" $U_{r}$ satisfies $U_{r} \leq\|u\|_{\infty}$ and its magnitude strongly depends on the distribution (and sign) of $W_{r}(x, t)$ in the vicinity of peak $|u|$.

Substituting (36) with $r=(q-2) / 2$ into (35) yields

$$
\begin{aligned}
\frac{\mathrm{d}}{\mathrm{d} t}\|u\|_{L^{q}} & \leq \frac{q-2}{\|u\|_{L^{q}}^{q-1}}\left(U_{(q-2) / 2}^{(q-2) / 2}\|p\|_{L^{2}}\left\||u|^{(q-2) / 2} \nabla|u|\right\|_{L^{2}}-\left\||u|^{(q-2) / 2} \nabla \mid u\right\|_{L^{2}}^{2}\right) \\
& \leq \frac{q-2}{\|u\|_{L^{q}}^{q-1}} U_{(q-2) / 2}^{q-2}\|p\|_{L^{2}}^{2}=\frac{(q-2) U_{(q-2) / 2}^{q-2}\|p\|_{L^{2}}^{2}}{\|u\|_{L^{q}}^{q}}\|u\|_{L^{q}},
\end{aligned}
$$

where Young's inequality has been used. It follows that

$$
\|u\|_{L^{q}} \leq\left\|u_{0}\right\|_{L^{q}} \exp \left\{(q-2) \int_{0}^{t} \frac{U_{(q-2) / 2}^{q-2}\|p\|_{L^{2}}^{2}}{\|u\|_{L^{q}}^{q}} \mathrm{~d} \tau\right\} .
$$

For a given $q \geq 3$, the integral on the right-hand side of (39) remains finite under a broad class of conditions on $U_{(q-2) / 2}$ and regularity follows. For example, with $q=3$ we have

$$
\int_{0}^{t} \frac{U_{1 / 2}\|p\|_{L^{2}}^{2}}{\|u\|_{L^{3}}^{3}} \mathrm{~d} \tau \leq c \int_{0}^{t} \frac{U_{1 / 2}\|u\|_{L^{4}}^{4}}{\|u\|_{L^{3}}^{3}} \mathrm{~d} \tau \leq c \int_{0}^{t} \frac{U_{1 / 2}\|u\|_{L^{6}}^{2}}{\|u\|_{L^{3}}} \mathrm{~d} \tau,
$$

where Calderon-Zygmund's and Hölder's inequalities have been used. Due to Sobolev's inequality $\|u\|_{L^{6}} \leq c\|\omega\|_{L^{2}}$, the right-hand side of (40) is finite as long as $U_{1 / 2} \leq c\|u\|_{L^{3}}$. Similarly, for $q=4$ we have

$$
\int_{0}^{t} \frac{U_{1}^{2}\|p\|_{L^{2}}^{2}}{\|u\|_{L^{4}}^{4}} \mathrm{~d} \tau \leq c \int_{0}^{t} \frac{U_{1}^{2}\|u\|_{L^{4}}^{4}}{\|u\|_{L^{4}}^{4}} \mathrm{~d} \tau=c \int_{0}^{t} U_{1}^{2} \mathrm{~d} \tau,
$$

which is finite as long as $U_{1} \leq c\|\omega\|_{L^{2}}$. For $q \geq 6$, we have

$$
\int_{0}^{t} \frac{U_{(q-2) / 2}^{q-2}\|p\|_{L^{2}}^{2}}{\|u\|_{L^{q}}^{q}} \mathrm{~d} \tau \leq c\left(\int_{0}^{t} \frac{U_{(q-2) / 2}^{2(q-2)}\|u\|_{L^{6}}^{4}}{\|u\|_{L^{q}}^{2 q}} \mathrm{~d} \tau\right)^{1 / 2}
$$

which is finite if $U_{(q-2) / 2} \leq c\|u\|_{L^{q}}$. In this case, the integrand on the right-hand side diminishes as $\|u\|_{L^{q}}$ increases, leading to saturated growth of $\|u\|_{L^{q}}$. 
3.3. Refined bounds. We now take a closer look at the negative correlation between $|u|$ and $|\nabla| u||$ and explore this feature in more detail. The results are summarised in the following theorems.

Theorem 4. Let $u$ and $p$ solve the Navier-Stokes equations (1). Given $q \geq 3$ as large as necessary, let $\Omega \subset \mathbb{R}^{3}$ be such that the following hypothesis, say (H1),

- $|u|>\left(\frac{c}{q-2} \frac{\|u\|_{L^{q}}^{2}}{\|p\|_{L^{2}}}\|\omega\|_{L^{2}}\right)^{1 /(q-2)}\|u\|_{L^{q}}$, for $x \in \Omega$;

- $|\widehat{u} \cdot \nabla| u|| \leq c\|\omega\|_{L^{2}}^{2}$, for $x \in \Omega$;

- $|u| \leq\left(\frac{c}{q-2} \frac{\|u\|_{L^{q}}^{2}}{\|p\|_{L^{2}}}\|\omega\|_{L^{2}}\right)^{1 /(q-2)}\|u\|_{L^{q}}$, for $x \notin \Omega$

holds. Then

$$
\|u\|_{L^{q}} \leq\left\|u_{0}\right\|_{L^{q}} \exp \left\{c q \int_{0}^{t}\|\omega\|_{L^{2}}^{2} \mathrm{~d} \tau\right\}
$$

and regularity follows.

Proof. By recalling (35) and invoking (H1) we obtain

$$
\begin{aligned}
\|u\|_{L^{q}}^{q-1} \frac{\mathrm{d}}{\mathrm{d} t}\|u\|_{L^{q}} & \leq(q-2) \int_{\mathbb{R}^{3}} p|u|^{q-2} \widehat{u} \cdot \nabla|u| \mathrm{d} x \\
& \leq c(q-2)\|\omega\|_{L^{2}}^{2} \int_{\Omega}\left|p \| u | ^ { q - 2 } \mathrm { d } x + c \frac { \| u \| _ { L ^ { q } } ^ { q } } { \| p \| _ { L ^ { 2 } } } \| \omega \left\|_{L^{2}} \int_{\mathbb{R}^{3}}|p\|\nabla \mid u\| \mathrm{d} x\right.\right. \\
& \leq c(q-2)\|\omega\|_{L^{2}}^{2}\|p\|_{L^{q / 2}}\|u\|_{L^{q}}^{q-2}+c\|u\|_{L^{q}}^{q}\|\omega\|_{L^{2}}^{2} \\
& \leq c(q-2)\|\omega\|_{L^{2}}^{2}\|u\|_{L^{q}}^{q}+c\|u\|_{L^{q}}^{q}\|\omega\|_{L^{2}}^{2},
\end{aligned}
$$

where the Calderon-Zygmund inequality has been used. By absorbing the second term on the right-hand side into the first term, we can write

$$
\frac{\mathrm{d}}{\mathrm{d} t}\|u\|_{L^{q}} \leq c q\|\omega\|_{L^{2}}^{2}\|u\|_{L^{q}}
$$

The theorem is proven upon integration.

Evidently, a singularity would necessarily require $q \rightarrow \infty$ in (H1). This corresponds to a complete overturn of the negative correlation between $|u|$ and $|\nabla| u||$. Interestingly, in that limit the assumption for $u$ outside $\Omega$ becomes $|u| \leq\|u\|_{\infty}$. Note that both $|\nabla p| \rightarrow \infty$ and $|p| \rightarrow \infty$ are required for a singularity, i.e. an overturn of the negative correlation between $|p|$ and $|\nabla p|$ as well.

Criteria similar to the above, i.e. hypothesis (H1), can be derived in the same manner. For example, one may replace the second condition in (H1) by $p \widehat{u} \cdot \nabla|u| \leq c|u|^{2}\|\omega\|_{L^{2}}^{2}$, for $x \in \Omega$, without altering the conclusion (43). This condition can be seen to be more optimal than its counterpart in (H1), due to the possibilities that $|p|$ may not peak at the same place as $|u|$ and that $p \widehat{u} \cdot \nabla|u|$ could be negative at low $p$. Finally, by taking into consideration the effects of both dissipation terms in (35), we have the following variation of (H1).

Theorem 5. Let $u$ and $p$ solve the Navier-Stokes equations (1). Given $q \geq 3$ as large as necessary, let $\Omega \subset \mathbb{R}^{3}$ be such that the following hypothesis, say (H2),

- $|u|>\left(\frac{c}{q-2} \frac{\|u\|_{L^{q}}^{2}}{\|p\|_{L^{2}}}\|\omega\|_{L^{2}}\right)^{1 /(q-2)}\|u\|_{L^{q}}$, for $x \in \Omega$;

- $p \widehat{u} \cdot \nabla|u|-\left.|\nabla| u\right|^{2}-\frac{|\nabla u|^{2}}{q-2} \leq c|u|^{2}\|\omega\|_{L^{2}}^{2}$, for $x \in \Omega$;

- $|u| \leq\left(\frac{c}{q-2} \frac{\|u\|_{L^{q}}^{2}}{\|p\|_{L^{2}}}\|\omega\|_{L^{2}}\right)^{1 /(q-2)}\|u\|_{L^{q}}$, for $x \notin \Omega$ 
holds. Then

$$
\|u\|_{L^{q}} \leq\left\|u_{0}\right\|_{L^{q}} \exp \left\{c q \int_{0}^{t}\|\omega\|_{L^{2}}^{2} \mathrm{~d} \tau\right\}
$$

and regularity follows.

Proof. Similar to (44), substituting (H2) into (35) yields

$$
\begin{aligned}
\|u\|_{L^{q}}^{q-1} \frac{\mathrm{d}}{\mathrm{d} t}\|u\|_{L^{q}} \leq & (q-2) \int_{\mathbb{R}^{3}} p|u|^{q-2} \widehat{u} \cdot \nabla|u| \mathrm{d} x-(q-2) \int_{\mathbb{R}^{3}}|u|^{q-2}|\nabla| u \|^{2} \mathrm{~d} x \\
& -\int_{\mathbb{R}^{3}}|u|^{q-2}|\nabla u|^{2} \mathrm{~d} x \\
\leq & c(q-2)\|\omega\|_{L^{2}}^{2} \int_{\Omega}|u|^{q} \mathrm{~d} x+c \frac{\|u\|_{L^{q}}^{q}}{\|p\|_{L^{2}}}\|\omega\|_{L^{2}} \int_{\mathbb{R}^{3}}|p\|\nabla \mid u\| \mathrm{d} x \\
\leq & c(q-2)\|\omega\|_{L^{2}}^{2}\|u\|_{L^{q}}^{q}+c\|u\|_{L^{q}}^{q}\|\omega\|_{L^{2}}^{2},
\end{aligned}
$$

which immediately implies (45). The proof is then completed upon integration. As in the previous case, a loss of regularity would necessarily require $q \rightarrow \infty$ in (H2).

\section{Concluding Remarks}

We have examined the pressure term that drives the dynamics of the velocity norm $\|u\|_{L^{q}}$, for $q \geq 3$, and derived several bounds for $\|u\|_{L^{q}}$. Regularity criteria have been deduced by requiring that these bounds remain finite.

The pressure term in the governing equation for $\|u\|_{L^{q}}$ has a certain degree of depletion of nonlinearity, owing to the negative correlation between $|u|$ and $|\nabla| u||$. Under viscous effects, this depletion is expected to give rise to relatively mild growth of $\|u\|_{L^{q}}$. However, these effects are not known to be capable of preventing an overturn of the favourable correlation between $|u|$ and $|\nabla| u||$. It is interesting to note that in the $n$-dimensional Burgers equation $(n \geq 1)$, where there is no pressure and $\|u\|_{L^{\infty}}$ is a priori bounded, a relatively weak dissipation mechanism represented by $(-\Delta)^{1 / 2}$ is sufficiently strong to regularize the dynamics [14].

Acknowledgment. This paper was presented at the workshop "Geometry and Fluids" (Clay Mathematics Institute, Oxford). C.V.T. would like to acknowledge stimulating discussions with the workshop participants, particularly J. Gibbon, G. Seregin, E. Titi, R. Kerr and M. Cullen. X.Y. was partially supported by an NSERC Discovery Grant. We thank the anonymous referees for helpful comments.

\section{REFERENCES}

[1] Ashurt Wm T, Kerstein A R, Kerr R M and Gibson C H 1987 Alignment of vorticity and scalar gradient with strain rate in simulated Navier-Stokes turbulence Phys. Fluids 30 2343-2353

[2] Beale J T, Kato T and Majda A 1984 Remarks on the breakdown of smooth solutions for the 3-D Euler equations Commun. Math. Phys. 94 61-64

[3] Beirao da Veiga H and Berselli L C 2002 On the regularizing effect of the vorticity direction in incompressible viscous flows Differential Integral Equations 15 345-356

[4] Berselli L C 2009 Some geometric constraints and the problem of global regularity for the Navier-Stokes equations Nonlinearity 22 2561-2581

[5] Berselli L C and Galdi G P 2002 Regularity criteria involving the pressure for the weak solutions to the Navier-Stokes equations Proc. Amer. Math. Soc. 130 3585-3595 
[6] Bos W J T and Rubinstein R 2013 On the strength of the nonlinearity in isotropic turbulence J. Fluid Mech. $733158-170$

[7] Caffarelli L, Kohn R and Nirenberg L 1982 Partial regularity of suitable weak solutions of the Navier-Stokes equations Commun. Pure and Applied Math. 35 771-831

[8] CaO C And Titi E S 2010 Sufficient conditions for the regularity to the $3 D$ Navier-Stokes equations Discrete Contin. Dyn. Syst. 26 1141-1151

[9] CaO C And Titi E S 2011 Global regularity criterion for the 3D Navier-Stokes equations involving one entry of the velocity gradient tensor Arch. Rational Mech. Anal. 202 919-932

[10] Chae D ANd LeE J 2001 Regularity in terms of pressure for the Navier-Stokes equations Nonlinear Analysis $46727-735$

[11] Chan C H And Yoneda T 2012 On possible isolated blow-up phenomena and regularity criterion of the 3D Navier-Stokes equation along the streamlines Methods Appl. Anal. 19 211-242

[12] Constantin P and Fefferman C 1993 Direction of vorticity and the problem of global regularity for the Navier-Stokes equations Indiana Univ. Math. J. 42 775-789

[13] Constantin P, Fefferman C and Majda A 1996 Geometric constraints on potentially singular solutions for the 3D Euler equations Commun. Partial Differential Equations 21 559-571

[14] Constantin P And Vicol V 2012 Nonlinear maximum principles for dissipative linear nonlocal operators and applications Geom. Funct. Anal. 22 1289-1321

[15] Deng J, Hou T And Yu X 2005 Geometric properties and nonblowup of 3D incompressible Euler flow Commun. Partial Differential Equations 30 225-243

[16] Deng J, Hou T And Yu X 2006 Improved geometric conditions for non-blowup of the 3D incompressible Euler equation Commun. Partial Differential Equations 31 293-306

[17] Donzis D A, Gibbon J D, Gupta A, Kerr R M, Pandit R and Vincenzi D 2013 Vorticity moments in four numerical simulations of the 3D Navier-Stokes equations J. Fluid Mech. 732 316-331

[18] Escauriaza L, Seregin G A and Sverák V $2003 L_{3, \infty}$-solutions of Navier-Stokes equations and backward uniqueness Uspekhi Mat. Nauk. 58 3-44

[19] Giga Y 1986 Solutions for semilinear parabolic equations in $L^{p}$ and regularity of weak solutions of the NavierStokes equations J. Differential Equations 62 186-212

[20] Gruji Z and Zhang Q S 2006 Space-time localization of a class of geometric criteria for preventing blow-up in the 3D NSE Comm. Math. Phys. 262 555-564

[21] Hou T Y AND LI R 2006 Dynamic depletion of vortex stretching and non-blowup of the 3-D incompressible Euler equations J. Nonlinear Sci. 16 639-644

[22] Kato T and Ponce G 1988 Commutator estimates and the Euler and Navier-Stokes equations Commun. Pure and Applied Math. 41 891-907

[23] Katz N and Pavlovic N 2002 A cheap Caffarelli-Kohn-Nirenberg inequality for the Navier-Stokes equation with hyper-dissipation Geom. Funct. Anal. 12 355-379

[24] Kida S and Kataoka M 1994 Vortex reconnection Annu. Rev. Fluid Mech. 26 169-189

[25] Kozono H and TAnuichi Y 2000 Limiting case of the Sobolev inequality in BMO, with application to the Euler equations Commun. Math. Phys. 214 191-200

[26] Kukavica I And Ziane M 2006 One component regularity for the Navier-Stokes equations Nonlinearity 19 453-469

[27] Kukavica I And Ziane M 2007 Navier-Stokes equations with regularity in one direction J. Math. Phys. 48 065203

[28] Ladyzhenskaya O A 1967 On uniqueness and smoothness of generalized solutions to the Navier-Stokes equations Zapiski Nauchn. Seminar POMI 5 169-185

[29] LeE J 2012 Notes on the geometric regularity criterion of 3D Navier-Stokes system J. Math. Phys. 53073103

[30] Meneveau C 2011 Lagrangian dynamics and models of the velocity gradient tensor in turbulent flows Annu. Rev. Fluid Mech. 43 219-245

[31] OhкітAni K 2009 Non-linearity depletion, elementary excitations and impulse formulation in vortex dynamics Geophys. Astro. Fluid Dyn. 103 113-133

[32] O'LEARY M 1998 Pressure conditions for the local regularity of solutions of the Navier-Stokes equations $J$. Differential Equations 12 1-9

[33] Prodi G 1959 Un teorema di unicità per el equazioni di Navier-Stokes Ann. Mat. Pura Appl. 48 173-182

[34] Saffman P G 1990 A model for vortex reconnection J. Fluid Mech. 212 395-402 
[35] Seregin G and Sverák V 2002 Navier-Stokes equations with lower bounds on the pressure Arch. Rational Mech. Anal. 163 65-86

[36] SERRin J 1962 On the interior regularity of weak solutions of the Navier-Stokes equations Arch. Rational Mech. Anal. 9 187-195

[37] Struwe M 1988 On partial regularity results for the Navier-Stokes equations Commun. Pure and Applied Math. $41437-458$

[38] TAO T 2009 Global regularity for a logarithmically supercritical hyperdissipative Navier-Stokes equation Anal. Partial Differential Equations 2 361-366

[39] VASSEUR A 2009 Regularity criterion for 3D Navier-Stokes equations in terms of the direction of the velocity Appl. Math. 54 47-52.

[40] ZHou Y 2002 A new regularity criterion for the Navier-Stokes equations in terms of the gradient of one velocity component Methods Appl. Anal. 9 563-578

[41] Zноu Y 2004 Regularity criteria in terms of pressure for the 3-D Navier-Stokes equations in a generic domain Math. Ann. 328 173-192

[42] Zhou Y 2005 On regularity criteria in terms of pressure for the Navier-Stokes equations in $\boldsymbol{R}^{3}$ Proc. Amer. Math. Soc. 134 149-156

[43] Zhоu Y 2005 A new regularity criterion for weak solutions to the Navier-Stokes equations J. Math. Pure Appl. 84 1496-1514

[44] Zноu Y 2005 Direction of vorticity and a new regularity criterion for the Navier-Stokes equations ANZIAM J. $46309-316$

[45] ZHOU Y 2005 A new regularity criterion for the Navier-Stokes equations in terms of the vorticity direction Monatsh. Math. 144 251-257

[46] Zhou Y And Pokorny M 2009 On a regularity criterion for the Navier-Stokes equations involving gradient of one velocity component J. Math. Phys. 50123514

[47] Zhou Y And Pokorny M 2010 On the regularity of the solutions of the Navier-Stokes equations via one velocity component Nonlinearity 23 1097-1107

Chuong V. Tran: School of Mathematics and Statistics, University of St Andrews, St Andrews KY16 9SS, United Kingdom

E-mail address: cvt1@st-andrews.ac.uk

Xinwei Yu: Department of Mathematical and Statistical Sciences, University of Alberta, Edmonton, AB, T6G 2G1, CANADA

E-mail address: xinweiyu@math.ualberta.ca 